\title{
LE FINANCEMENT DES CAMPAGNES POLITIQUES: UN DIALOGUE AVEC LA THEORIE D'OLIVER WILLIAMSON
}

\author{
FINANCING THE ELECTION CAMPAIGNS : \\ A DIALOGUE WITH OLIVER WILLIAMSON'S THEORY
}

\author{
FINANCIACIÓN DE LAS CAMPAÑAS ELECTORALES: \\ UN DIÁLOGO CON LA TEORÍA DE OLIVER WILLIAMSON
}

\section{FINANCIAMENTO DAS CAMPANHAS ELEITORAIS : UM DIÁLOGO COM A TEORIA DE OLIVER WILLIAMSON}

\begin{abstract}
Giovani Ribeiro Rodrigues AlVes
https://orcid.org/0000-0002-9278-9541 / http://lattes.cnpq.br/2325592312806024 / giovanirralves@uol.com.br Unibrasil Centro Universitário - UNIBRASIL Curitiba, PR, Brasil.

marcia Carla Pereira Ribeiro

https://orcid.org/0000-0001-7540-5406 / http://lattes.cnpq.br/1514127912243020 / mcarlaribeiro@uol.com.br Universidade Federal do Paraná - UFPR Pontifícia Universidade Católica do Paraná - PUC Curitiba, PR, Brasil.
\end{abstract}

\begin{abstract}
RÉSUMÉ
Le financement est indispensable aux campagnes politiques et les moyens autorisés par la loi peuvent influencer le résultat des élections. En ce moment, un grand débat est en cours au Brésil, à la suite des scandales de corruption qui ont bouleversé la société PETROBRAS, plusieurs agents politiques et de grands entrepreneurs nationaux, relativement au financement des partis politiques et des élections. La Cour Suprême brésilienne, à partir d'une interprétation de la Constitution, en 2015, a jugé l'illégalité de donation de personnes morales. Ainsi, à partir de l'élection qui a eu lieu au Brésil en octobre 2018, le financement est majoritairement garanti à partir de fonds financiers spécifiques de l'État, autorisées des donations de personnes physiques, limité à un certain pourcentage du salaire minimum fixé annuellement par l'État. La proposition que j'aimerais présenter pour le débat est d'analyser, à partir de la théorisation d'Oliver Williamson à propos de formes de gouvernance (contrat, marché et firme), des possibilités de financement plus compatibles avec le gouvernement ouvert.
\end{abstract}

Mots-clés: Élections ; Financement ; Gouvernance ; Nouvelle Économie Institutionnelle

\section{RESUMO}

O financiamento é indispensável às campanhas eleitorais e os meios autorizados pela lei podem influenciar no resultado das eleicões. Atualmente há um importante debate no Brasil, após os escândalos de corrupção que atingiram a empresa PETROBRÁS, vários agentes políticos e grandes empresários nacionais, com relação ao financiamento dos partidos políticos e das eleições. O Supremo Tribunal Federal, a partir de uma interpretação da Constituição Federal, no ano de 2015, declarou a ilegalidade de doações realizadas por pessoas jurídicas. Desta forma, a partir das eleições que ocorreram em outubro de 2018 no Brasil, o financiamento é majoritariamente garantido a partir de fundos financeiros específicos de titularidade do Estado, sendo admitida a doação de pessoas físicas, limitadas a um certo percentual do salário mínimo fixado anualmente. A proposição do artigo é trazer a debate, a partir da teoria desenvolvida por Oliver Williamson em relação às estruturas de governança (contrato, mercado e firma), as possibilidades de financiamento mais compatíveis com o governo aberto.

Palavras-chave: Eleições; Financiamento; Governança; Nova Economia Institucional. 


\begin{abstract}
Funding is indispensable to election campaigns and the ways authorized by law can influence the outcome of the elections. There is currently an important debate in Brazil, following the corruption scandals that hit PETROBRÁS, various political agents and major national entrepreneurs, regarding the financing of political parties and elections. The Supreme Court, based on an interpretation of the Federal Constitution, in 2015, declared the illegality of donations made by legal entities. Consequently, from the elections held in October 2018 in Brazil, the financing is mainly guaranteed from specific state-owned financial funds, and the donation of individuals is allowed, limited to a certain percentage of the minimum wage set annually. The purpose of the article is to bring to the debate, based on the theory developed by Oliver Williamson regarding the governance structures (contract, market and firm), the financing possibilities more compatible with open government.
\end{abstract}

Keywords: Elections; Financing; Governance; New Institutional Economics.

\title{
RESUMEN
}

La financiación es indispensable para las campañas electorales y los medios autorizados por la ley pueden influir en el resultado de las elecciones. Actualmente hay un importante debate en Brasil, después de los escándalos de corrupción que golpearon a PETROBRÁS, varios agentes políticos y grandes empresarios nacionales, con respecto al financiamiento de partidos políticos y elecciones. La Corte Suprema, basada en una interpretación de la Constitución Federal, en 2015, declaró la ilegalidad de las donaciones hechas por personas jurídicas. Por lo tanto, a partir de las elecciones celebradas en octubre de 2018 en Brasil, el financiamiento está garantizado principalmente por fondos financieros estatales específicos, y se permite la donación de individuos, limitada a un cierto porcentaje del salario mínimo establecido anualmente. El propósito del artículo es llevar al debate, basado en la teoría desarrollada por Oliver Williamson sobre las estructuras de gobierno (contrato, mercado y firma), las posibilidades de financiamiento más compatibles con el gobierno abierto.

Palabras clave: Elecciones; Financiación; Gobernanza; Nueva Economía Institucional.

\section{SUMÁRIO}

INTRODUCTION; 1 TRANSPARENCE : UNE INVITATION À LA PARTICIPATION; 2 LE MYTHE DE LA RATIONALITE DE L'ELECTEUR; 3 LES FORMES DE GOUVERNANCE ; 4 LES BENEFICES DE LA COOPERATION; 5 LA GOUVERNANCE DANS LES MODELES DE FINANCEMENT DES CAMPAGNES POLITIQUES ; CONCLUSION ; BIBLIOGRAFIE.

\section{INTRODUCTION}

Il est absolument clair que de plus en plus les citoyens perdent leur intérêt relativement au processus politique d'élection pour l'administration au niveau du Pouvoir Exécutif et du Pouvoir Législatif. Quand on se demande la raison pour ce manque d'intérêt, plusieurs arguments peuvent être envisagés.

Premièrement, il est possible que l'électeur, à partir de son analyse rationnelle, ait pris conscience de la limitation de son accès aux résultats effectifs, associés au mandat de celui qui a été élu, ainsi comme d'une absence d'informations accessibles et fiables. Ce que j'appelle le défaut d'information post élection. 
À la suite, il se peut que l'analyse rationnelle soie tournée à la perception que ni le résultat de l'élection, ni le résultat de l'action de l'agent public n'auraient un vrai ou important impact vis-à-vis de la vie de l'électeur.

En plus, il y a la perception que ce n'est pas le vote individuel qui fera la différence puisque le résultat des élections peut être facilement associé à l'action des groupes d'intérêt plus organisés et plus riches, dont les intérêts seront plus tard surnommés intérêt public, et qui représentent les intérêts d'un petit groupe prévalent.

Indépendamment de la théorisation diffusée au monde à propos de l'adoption du modèle politique démocratique et des évidences empiriques de ses avantages comparativement aux systèmes dites autocratiques, peut-être que nous sommes en retard pour ce qui concerne la constatation que les groupes d'intérêt agissent par moyen du lobby et de l'impuissance de n'importe quelle initiative consacrée à leurs éliminations.

Quand c'est une question d'administration, n'importe quelle organisation (soit une entreprise ou le gouvernement) aura la charge de choisir la meilleure stratégie de gestion, ce que, selon la Nouvelle Économie Institutionnel-NEI, implique la recherche des besoins organisationnels au marché, l'établissement de contrats dits relationnels ou l'internalisation des coûts associés à ces besoins.

L'article part d'une idée centrale, une proposition d'application des prémisses de la NEI, spécialement à partir de Williamson, relativement au financement des campagnes politiques, ayant pour but de proposer que l'instrument associatif et par conséquent contractuel, peut être envisagé comme une nouvelle façon de financement politique qui peut contribuer à la configuration d'une nouvelle face pour la déjà ancienne dame nommée démocratie.

\section{TRANSPARENCE : UNE INVITATION A LA PARTICIPATION}

Imaginons qu'il soit possible d'accompagner jus in time, pas seulement les propositions exposées par le candidat avant et pendant la période électorale, mais aussi ses actions post élection. D'une façon simplifiée, accessible et claire afin de rendre possible une comparaison entre ce qui a été annoncé comme programme politique et ce qui a été réalisé ou pas mis en pratique.

C'est possible de soupçonner qu'un système numérique s'en charge, en envoyant aux électeurs de rapports périodiques, ainsi comme une communication automatique à l'agent politique du contenu du rapport et la relation de ceux qui ont décidé d'avoir accès à ce rapport périodique. 
Quelques apports théoriques considèrent que la technologie blockchain peut rendre possible ce modèle de transparence, puisqu'elle permettrait une relation peer-to-peer qui n'a pas besoin des agents intermédiaires qui pourraient produire n'importe quelle interférence au sein des informations ainsi comme elles ont été construites ${ }^{1}$. Cela veut dire, la fiabilité des systèmes de contrôle serait agrandie par rapport à ce qui est disponible de nos jours. La technologie blockchain est une « solution inédite, décentralisée, sécurisée et transparente, permet de stocker, d'échanger, d'authentifier et de vérifier des informations pour un coût faible et supporté par l'utilisateur, donc sans un tiers garant $»^{2}$.

En principe, si on réduit les coûts de transaction ${ }^{3}$ (dans le cas analysé il est possible d'être considéré le coût d'avoir accès à l'information, du temps nécessaire à la compréhension des données disponibles, leurs potentiels impacts, surtout relativement aux aspects plus proches de ce qui concerne plus directement aux intérêts personnel), on favorise l'action de surveillance qui va probablement approcher l'électeur de l'agent politique de façon à l'influencer à une action compatible avec les propositions de campagne.

Nous commençons à nous rendre compte de ces possibilités à partir de l'influence des réseaux sociaux dans le résultat de quelques élections présidentielles, c'est le cas au Brésil, aux États-Unis et aussi en France. Pas seulement l'accès des candidats à des données des électeurs à partir des outils numériques, ainsi comme l'utilisation des réseaux sociaux ont influencé les résultats des élections ${ }^{4}$.

Maintenant il est possible d'imaginer que ces outils soient associés à la surveillance des élus, dans un contexte d'adoption d'une attitude participative des électeurs, de façon à ce qu'ils puissent s'engager vis-à-vis de leur choix, ou qu'une organisation dont ils participent puisse accomplir cette fonction.

La participation, en dehors du contrôle, pourra être visible aussi au niveau du financement des partis politiques.

1 TAPSCOTT \& TAPSCOTT, 2018, p. 5: “This has never happened before - trusted transactions directly between two or more parties, authenticated by mass collaboration and powered by collective self-interests, rather than by large corporations motivated by profit."

2 LOLOUP, L. BLOCKCHAIN: La Revolution De La Confiance. Paris, Eyrolles, 2018.p.20.

3 Le coût de transaction est le coût de négociation, associé aux échanges de biens ou de services et qui a son origine liée aux imperfections du marché. Il est directement associé à la décision de gouvernance entre produire ou acheter un bien ou un service. COASE, 1960, pp. 1- 44.

4 LESPAGNOL, J. L'influence de l'Internet sur les candidats. In : L'Internet et la démocratie numérique [en ligne]. Perpignan : Presses universitaires de Perpignan, 2016 (généré le 06 décembre 2018). [http://books.openedition.org/pupvd/2770]. ISBN : 9782354122980. DOI : 10.4000/books.pupvd.2770 (consulté le 2 décembre 2018). 
Avant d'y arriver, quelques notes à propos de la rationalité de l'électeur méritent d'être considérées.

\section{LE MYTHE DE LA RATIONALITE DE L'ELECTEUR}

L'électeur a très peu de rationalité en tête quand il vote. Ou, peut-être, c'est justement parce qu'il est rationnel qu'il se rend compte que son vote n'aura aucune importance dans le résultat électoral. Voilà la raison pour laquelle le taux d'abstention ne s'arrête pas d'agrandir dans les pays où le vote n'est pas obligatoire, la même chose pour ceux qui forcent le vote ${ }^{5}$.

Au Brésil, c'est assez fréquent d'entendre dire que les citoyens ne votent pas correctement, qu'ils choisissent très mal et, puisque l'électeur est ignorant, des erreurs systématiques ont comme résultat visible la qualité des politiciens. Par conséquent, ou l'électeur est tenu comme rationnellement ignorant ou il se trompe quand il vote et c'est la raison pour laquelle nous avons de mauvais politiciens ${ }^{6}$.

Mais ce n'est pas si simple que ça. Un système démocratique n'est pas uniquement caractérisé par la possibilité de suffrage universel. Il se conditionne à d'autres caractéristiques qui ont été mises en relief par Downs ${ }^{7}$ : le choix d'un seul parti politique (ou d'une coalition) ; élections périodiques et définies, électeurs qualifiés à voter et les candidats qui n’ont pas été élus ne doivent pas agir de façon à déranger l'action des élus.

À part ces conditions, il faut encore envisager l'absence de rationalité quand on imagine qu'un politicien soit moins auto-intéressé qu'un autre citoyen. Que, au moment où lui a été accordée une fonction publique, il laissera complètement de côté ses prétentions personnelles.

Comment résoudre ces idiosyncrasies du système démocratique ? Peut-être commencer par l'acceptation de l'idée que notre système politique a des imperfections qui méritent d'être corrigées. Il n'est plus le temps de fermer les yeux devant le manque de légitimité de quelques

5 En France, pour l'élection du président Macron, le taux d'abstention est le plus haut de l'histoire de France, depuis 1969, 25,44\% [https://www.lci.fr/]. Pas moins remarquable la quantite de votes nul ou blanc, 11,47\%. Au Brésil, pour le président élu Bolsonaro, ont été $20,3 \%$ d'abstention [https://g1.globo.com/] et 9,5\% de votes nul ou blanc [https://g1.globo.com/] (consultés le 2 décembre 2018).

6 KANAYAMA, Rodrigo. O mito do eleitor racional. in : Análise Econômica do Direito: Justiça e Desenvolvimento. M. C. P. RIBEIRO, V. H. DOMINGUES, V. KLEIN, Vinícius (dir.), Curitiba, Editora CRV, 2016, pp.23-26.

7 COASE, Ronald ; DOWNS, A. Uma Teoria Econômica da Democracia, São Paulo, Edusp, 1999. 
autorités élues. Légitimité que peut être admise comme « la capacité du système politique créer et maintenir la croyance dans le sens où les institutions représentent ce qu'il y a de mieux pour la société ${ }^{8}$.

Les citoyens sont de plus en plus réfractaires à la figure de l'autorité, au coût des gouvernements et aux mesures d'austérité qui atteignent les bénéfices sociaux qui leur ont été accordés auparavant.

Les nouvelles technologies peuvent améliorer la perspective de l'avenir et il faut y permettre cette amélioration puisque, jusqu'à maintenant, il nous est impossible d'indiquer une suggestion d'abandon du système démocratique par un autre système prouvé plus efficient.

Habituellement les systèmes autocratiques ont de bons résultats économiques et sociaux au premier moment, mais ils arrêtent aisément leurs performances à la suite?

Les mécanismes de contrôle des résultats des actions politiques permettraient de changer la base de la relation de l'électorat et des pouvoirs publics. Pourtant, l'excès d'information et la prévalence de l'inclination individuelle peuvent rendre très peu intéressant le choix pour l'information et la surveillance des agents publics. D'où l'idée de ne pas considérer la quantité d'information, mais la façon comme elle est organisée et arrive à tous ceux qui s'intéressent, spécialement quand il y a une organisation représentative des électeurs.

Les technologies numériques appliquées aux actions gouvernementales représentent une option envers la reconstruction des pratiques démocratiques établies sur la base de la rationalité de l'électeur. Une autre possibilité peut être associée à un grand changement positionnel : de la position individuelle de l'électeur à la position associative.

\section{LES FORMES DE GOUVERNANCE}

WILLIAMSON ${ }^{10}$ a coordonné une célèbre recherche à propos des éléments qui sont à l'origine du choix des organisations par rapport à leurs achats de services ou de biens. Quand l'homme d'affaires ou l'administrateur public décide ce qui leur faut pour accomplir les objectifs de leurs affaires, ils ont la possibilité de chercher au marché les options disponibles. Quand la voie adoptée est celle du marché, ils n'ont aucune assurance à propos de la possibilité de réalisation de

8 TAPSCOTT, D.' ; TAPSCOTT, A. BLOCKCHAIN REVOLUTION: HOW THE TECHNOLOGY BEHIND BITCOIN AND OTHERS CRYPTOCURRENCIES IS CHANGING THE WORD. NEW YORK, PORTFOLIO/PENGUIN, 2018, p. 201. 9 SEN, Amartya. Desenvolvimento Como Liberdade. 4. Ed. São Paulo, Companhia Das Letras, 2010. 10 WILLIAMSON, Oliver. The Economic Institutions Of Capitalism: Firms, Markets, Relational Contracting, New York, The Free Press, 1985. 
nouvelles affaires avec le partenaire choisi au marché, ni stabilité de prix, ni garantie de qualité par rapport au besoin de l'organisation.

Il existe aussi la possibilité de choisir un contrat avec un partenaire habituel en utilisant des contrats relationnels qui sont les contrats plus directement associés à l'objet de l'activité professionnel de l'entreprise, dépourvus du caractère éventuel, contrats de moyen ou long terme. Ce genre de contrat a pour but d'assurer au proposant qu'il ait exactement ce dont il a besoin, dans la quantité nécessaire et selon les caractéristiques contractées.

Ou, l'organisation peut considérer qu'il sera plus efficient d'internaliser la production des biens et services, avec le coût qui accompagne cette décision, mais aussi avec l'addition du caractère hiérarchique. Ça veut le dire, les négociations ne seront plus entre fournisseur et acheteur, ou entre vendeur et acheteur, mais entre patrons et travailleurs.

Voilà une approche très synthétique des structures de gouvernance en WILLIAMSON. Il a constaté aussi que l'entrepreneur va choisir parmi toutes les possibilités, celle qui, à son avis, sera compatible avec la réduction des coûts.

Les facteurs qui vont influencer la définition de la structure de gouvernance sont liés à la spécificité de ce qui va être l'objet de négociation, le comportement du contractant et la fréquence des négociations. Analysés chacun de ces facteurs, la décision sera mise en place. Marché, contrats ou internalisation, voilà les options disponibles.

Plus le bien ou le service est spécifique, plus augmente la tendance au choix ou d'une part, de réalisation de contrats relationnels, ou, d'autre part, d'internaliser la production. Entre les deux possibilités, normalement sera considérée l'extension du risque du contractant adopter des actions opportunistes par rapport à son cocontractant. Si l'opportunisme devient un problème, et par conséquent, un élément d'augmentation des coûts, l'internalisation peut se montrer la meilleure option.

\section{LES BENEFICES DE LA COOPERATION}

Dans cet article, ont été déjà exposées quelques réflexions à propos de l'insuffisance du vote comme une attractive à l'engagement du citoyen, considéré dans son aspect individuel. Pourtant, il n'y a pas comment présenter de façon incontestable un autre modèle qui puisse remplacer la démocratie qui légitime le pouvoir public à partir du suffrage.

D’autre part, les nouvelles technologies numériques peuvent offrir (et quelques-unes sont déjà disponibles) des plateformes plus démocratiques, dans le sens de facilitation de l'expression 
du choix politique et aussi en offrant la possibilité des électeurs de surveiller l'action politique, inclus la destination des ressources financières rendues possibles en raison de la tribulation.

Nous avons invoqué aussi, comme argument, la prévalence de l'influence de groupes d'intérêt plus organisés sur les décisions dites politiques, ou d'intérêt public. Aucune allusion ici aux conduites illicites, ce sont, simplement, les règles du jeu. Cela veut dire, nos institutions formelles et informelles établissent les comportements acceptables et répudient ceux qui ne le sont pas. Les institutions doivent être des instruments de réduction des coûts, puisque, quand un certain comportement est imposé ou interdit, par une loi, par exemple, le pouvoir soulevé de la personne se justifie dans la mesure où l'existence de la loi soit plus efficace que l'ouverture à la décision personnelle ou à la libre négociation privée. Les institutions doivent être efficientes pour être maintenues ${ }^{11}$.

L'efficacité des institutions électorales est en chèque. En parallèle, la technologie blockchain peut offrir quelques outils soit au niveau de la gestion de l'entreprise, soit au niveau de l'administration publique.

La concrétisation d'affaires sans intermédiaires, peer to peer, peut, en théorie, améliorer les opportunités d'introduction au marché des nouveaux agents économiques, puisque quelques barrières d'accès au marché peuvent être dégagées. Par exemple, l'intermédiaire financier (les banques) qui peut avoir sa fonction remplacée par une stratégie faisant appel au crownfunding ou aux fintechs, ou les coûts d'enregistrement sous responsabilité de tiers, puisque le blockchain fonctionne aussi comme une archive énorme, impénétrable et décentralisée.

Dans le domaine de l'administration publique, pour ce qui concerne le processus d'élection, c'est déjà assez connu dans divers systèmes électoraux, l'introduction du système de financement privé à partir des donations collectives appelées crownfunding, qui permettent à plusieurs donateurs (restreins normalement à des limites individuelles) de participer aux coûts des campagnes politiques. Nous sommes ici en face d'une situation d'action coopérative.

La coopération est aussi essentielle à l'efficacité des affaires qu'à l'efficacité au niveau gouvernementale. Pourtant, la conscience de cette stratégie n'est pas nécessairement intégrée au quotidien, ni des agents économiques, ni du citoyen en général.

Il faut mener la conscience individuelle à la compréhension des bénéfices potentiels qui peuvent être associés à la collaboration. Elle peut diminuer sensiblement les situations de litige (puisque l'action des agents sera orientée vers un seul sens, de façon à satisfaire les deux profits),

11 NORTH, D. C. Economic Performance Through Time. In: The American Economic Review, 1994, Pp. 359368. 
elle permet une allocation plus efficace de droits et de biens envisagés, puisqu'elle rend possible une utilisation partagée, ayant par conséquent une exploitation supérieure des excédents.

Les jeux de collaboration, pour les actions répétitives, sont associés aux meilleurs résultats $^{12}$. En droit des sociétés, il est assez commun que les professeurs expliquent à leurs étudiants que les agents économiques peuvent choisir d'organiser une société commerciale parce qu'ils considèrent que la possibilité de succès sera élargie. L'expectative est que la collaboration puisse renforcer les objectives, soit par l'association de capital, soit par celle de travail. Et, les institutions, ça veut dire dans ce cas les lois qui disciplinent les sociétés commerciales, prévoient déjà le devoir de versement du capital social suscrit. Par conséquent, la coopération ou la collaboration peut simplement dériver d'une initiative privée ou peut être induite par la voie législative.

De toute façon, il y a quelques comportements qui peuvent stimuler la coopération et agir dans le sens de préservation de cette stratégie. AXELROD considère qu'il ne faut pas être jaloux par rapport à la situation de l'autre personne ; ni être ce qui échoue le premier ; il faut adopter une attitude de collaboration ou pas comme une réponse à la joué précédente de l'autre ; et, il finalise, il ne faut pas être trop intelligent ${ }^{13}$.

En plus, il y a quelques mesures pas compliquées qui peuvent contribuer pour la diffusion de la collaboration comme une stratégie. Si le problème est excessivement complexe, il vaut mieux le découper. Les relations qui travaillent avec une perspective de future ou répétition sont plus compatibles avec la collaboration, cela veut dire qu'il est très intéressant d'élargir la durée des relations, de façon à convaincre l'autre et à se convaincre dans le sens que les expectatives avec l'avenir soient plus intéressantes que les résultats immédiats. C'est très important aussi de promouvoir une éducation et une culture vers la collaboration.

Une attitude de coopération, à part les options d'engagement et de surveillance par rapport aux choix politiques, est essentielle à ce qui sera objet de proposition dans cet article : un échange dans le modèle de financement des campagnes politiques.

12 AXELROD, Robert. The Evolution Of Cooperation. New York, Basic Books, 1984. 13 Ibidem, p.110. 


\section{LA GOUVERNANCE DANS LES MODELES DE FINANCEMENT DES CAMPAGNES POLITIQUES}

À nos jours, normalement le financement des campagnes électorales et des partis politiques est basé sur l'apport gouvernemental (public) et sur l'apport privé. Pour ce qui concerne l'apport privé quelques systèmes acceptent des dons de personnes physiques et de personnes morales, d'autres seulement ceux de personnes physiques, soumis, très fréquemment, a des limites.

Pour avoir une idée, le financement public des campagnes est adopté en Allemagne, en Espagne, aux États-Unis, en France, en Italie, au Japon et au Brésil, par contre, il est interdit en Angleterre. Pour ce qui concerne les dons électoraux ayant comme origine les entreprises, ils sont acceptés en Espagne, en Italie et en Angleterre ; avec quelques conditionnant aux États-Unis et au Japon ; sont interdits en France et au Brésil. Par rapport à l'origine des dons, le financement provenant de l'étranger n'est pas accepté, parmi les huit pays analysés, excepté en Angleterre et en Italie. Relativement à la publicité des comptes, elle est garantie aux États-Unis, en France, en Italie et au Brésil. La fixation des limites pour les dons privés existe aux États-Unis, en France, en Italie et au Brésil. Le contrôle des comptes n'est pas assuré au Japon ${ }^{14}$.

Il est incontestable que les partis politiques ont besoin d'argent pour soutenir leurs candidats et, par conséquent, leurs propositions. Il est aussi incontestable que les citoyens ont l'argent et le potentiel intérêt à soutenir les candidats. Comme résultat prévisible, il y a un lien entre les dépenses de campagne et les résultats électoraux ${ }^{15}$, même si c'est impossible d'affirmer que les candidats qui ont été le plus bénéficiés avec les dons seront nécessairement élus ${ }^{16}$.

Il y a, en addition, un fort risque à ce que les donateurs aient une position avantageuse aux futurs rapports économiques avec l'administration soit par le biais de marchés publics ou encore de certaines politiques publiques et de règlementations. Voilà ce qui est appelé le risque de la capture de l'administration publique par les affaires.

Ça ne veut pas dire qu'il s'agit des actions illégales mais, cette influence sera ajoutée à l'opinion publique, aux orientations des partis et aux lobbies, comme un autre élément qui peut

14 BACKES, A. L. Financiamento Partidario E Eleitoral: Alemanha, França, Portugal E Espanha. Congres National. Assessorie Legislative. 2013, [Http://Www2.Camara.Leg.Br/Atividade-Legislativa/Estudos-ENotas-Tecnicas/Publicacoes-Da-Consultoria-Legislativa/Areas-Da-Conle/Tema3/2012_22272.Pdf] (Consulte Le 10 Octobre 2018).

15 lbidem.

16 Opus Citatum. 
influencer les décisions par rapport, par exemple, a une certaine politique publique. Au moins, quand il y a pleine accessibilité aux rapports des dons, les citoyens peuvent utiliser les informations comme instrument d'analyse du mérite des choix publics. « Lorsque quelques intérêts puissants s'accaparent l'action politique, les règles sont susceptibles d'être faussées en faveur de quelques-uns, ce qui peut entrainer l'adoption de politiques publiques allant à l'encontre de l'intérêt général ${ }^{17}$.

L'imbrication entre le financement des élections et le marché public est à la base des scandales de corruption qui continuent à être en évidence au Brésil. Les scandales sont assez diffusés aux multiples niveaux de l'organisation administrative à l'échelon national, régional et local, mais les plus emblématiques sont associés à l'entreprise d'économie mixte du pétrole, la PETROBRAS. Les contrats hors de prix identifiés ont eu pour but quelques fois d'enrichir les agents politiques, quelques fois ont été destinés aux partis politiques ${ }^{18}$. Malheureusement la réalité brésilienne a prouvé pas seulement le risque de la capture, mais aussi les dangers du capitalisme quand il devient un capitalisme de connivence, caractérisé par une proximité exagérée entre l'État et les entreprises ${ }^{19}$.

C'est justement là la justificative à l'interdiction des dons électoraux des entreprises à partir de l'élection de 2018 au Brésil.

Pour l'OCDE, il y a quelques principes qui peuvent favoriser l'amélioration du système démocratique vers la croissance inclusive. L'objectif global étant de promouvoir de règles du jeu équitable, la proposition est de l'acceptation du financement par le biais des contributions publiques directes et indirectes, associée à des financements privés, à l'application des plafonds de dépenses et à la limitation d'accès privilégié aux ressources publiques. Les financements indirects prévus comprenant les exonérations fiscales, l'accès subventionné aux médias et d'autres possibilités. La suggestion est de l'interdiction des financements privés provenant d'intérêts étrangers, des dons d'entreprises, de syndicats et de la limitation des dons anonymes ${ }^{20}$.

17 BACKES, A. L. Financiamento Partidário e Eleitoral: Alemanha, França, Portugal e Espanha. Congrès National. Assessorie Legislative. 2013, [http://www2.camara.leg.br/atividade-legislativa/estudos-e-notastecnicas/publicacoes-da-consultoria-legislativa/areas-da-conle/tema3/2012_22272.pdf] (consulté le 10 octobre 2018).

18 BOURCIER, N. ; GATINOIS, C. BRESIL : Tout Comprendre A L'operation « Lava Jato ». 2017. [HTTPS: / /WWW.LEMONDE.FR/AMERIQUES/ARTICLE/2017/03/26/AFFAIRE-PETROBRAS-RETOUR-SUR-LESTROIS-ANNEES-QUI-ONT-MARQUE-LE-BRESIL_5100932_3222.HTML].

19 ZINGALES, Luigi. Um Capitalismo Para O Povo: Reencontrando A Chave Da Prosperidade Americana, São Paulo, Bei Comunicação, 2015.

20 OECD (2017), Le financement de la démocratie: Financement des partis politiques et des campagnes électorales et risque de capture de l'action publique, Examens de l'OCDE sur la gouvernance publique, OECD Publishing, Paris. p. 31. https://doi.org/10.1787/9789264263994-fr. 
Cependant, la plupart des mesures suggérées peuvent être contournées par des stratégies assez simples. La limitation des dons peut donner lieu à la diversification formelle des donateurs ; l'interdiction de financement privé provenant d'intérêts étrangers - dans un monde d'économie globalisée - peut être relativisée à partir du concept d'intérêts étrangers par rapport à l'intérêt interne du pays.

Pour ce qui concerne les dons des entreprises et des syndicats, l'article suggère une vision particulière.

Le financement des campagnes électorales et des partis politiques doit parvenir des sources pour que ce soit moins difficile la recomposition du capital investi. Quand le financement est public, les sources publiques (limitées) seront retirées de la caisse générale, la même qui garantit la provision des services publics, par exemple. Il n'y a aucune garantie que l'argent dépensé (direct ou indirectement) avec les partis politiques et les campagnes électorales retourneront à sa source. Peut-être que dans le sens de la promotion d'une stabilité sociale qui puisse favoriser l'environnement institutionnel social et économique.

Mais, il y a surement d'autres agents plus directement favorisés par les résultats des élections, pas dans le sens d'obtenir des avantages injustifiables par rapport à la croissance inclusive, plutôt dans le sens de l'expression d'une option politique et économique que, à leur avis, devrait prévaloir, parce que cela les intéresse, parce que c'est convenant pour les affaires, ou parce que c'est l'expression d'une idéologie. Et, surtout, l'argent destiné au financement des élections ne sera pas retiré de la caisse générale des services publics.

Cette constatation est vraie pour n'importe quel pays, mais spécialement relevant pour les pays non développés. C'est assez dramatique d'imaginer qu'un pays très déficitaire en qualité de vie de la majorité de sa population puisse verser ses ressources limitées au financement des campagnes électorales.

Il nous reste les options privées de financements : l'argent des personnes physiques et l'argent des personnes morales.

Il existe une vision qui se méfie des relations entre l'administration publique et le monde des affaires et, des expériences pratiques qui confirment la crainte. Pourtant, l'abonnement public ne semble pas être la meilleure solution.

Même si le rapport de l'OCDE offre une position antagonique, il faut penser sérieusement au financement électoral par la voie des organisations relationnelles.

A partir des structures de gouvernance en WILLIAMSON, c'est possible d'établir une relation entre elles et les systèmes de financement des campagnes. Le marché peut être associé au 
financement privé libre et sans limitation par donateurs, comme en Angleterre. Dans ce système les partis et les candidats auront accès exclusivement aux ressources privées, sans distinction parmi les personnes morales ou physiques. S'il est possible de distorsions car les candidats les plus riches aurons plus accès aux médias et d'autres stratégies de communication avec le potentiel électorat, il existe toujours la possibilité de limitation non pas concernant les dons, mais pour ce qui concerne les dépenses des partis et des campagnes. Cette limitation pourrait réduire la différence entre les candidats, imposant des limites de coûts, suivi nécessairement de la surveillance des comptes.

Par contre, plusieurs pays suivent la tendance de fixation sur le modèle de financement public. Cette méthode s'approche de la structure d'internalisation en WILLIAMSON et en COASE (firme). L'État (organisation) va payer les campagnes et être la principale source de maintien des partis. La solution pour la demande de l'organisation (avoir des agents élus à occuper les fonctions publiques) sera internalisée. Comme dans le cas de l'entreprise, la recherche au marché est remplacée par des décisions hiérarchiques : combien sera destiné à chaque parti, la définition du critère de partage et d'autres décisions.

L'État ici apparaît comme la personne morale qui, à partir d'une position hiérarchique absolue, au nom de l'intérêt public, prend les décisions et internalise les coûts. Ici, à notre avis, les défauts les plus évidents sont liés à l'impossibilité de définition du contenu de ce qui sera admis comme expression de l'intérêt public (et qui sera son porte-parole) et, plus grave, la tendance d'éloignement des citoyens et des entreprises qui ne sont pas stimulés à sacrifier une partie de leurs disponibilités financières à donner support à un projet politique. Voilà un terrain fertile pour l'absentéisme électoral.

Une suggestion n'a pas été jusqu'à maintenant confrontée aux précédentes. Il s'agit de la délégation du pouvoir de financement électoral à des organisations relationnelles, dans une approche que Williamson a défini en relation aux contrats relationnels.

Les contrats relationnels, comme il a été déjà exposé, sont des contrats caractérisés par des négociations qui ne seront ni éventuelles ni du genre d'affaire où les intérêts des agents économiques viennent en sens opposé (par exemple, pour le vendeur le prix le plus haut possible, pour l'acheter le plus bas). Contrairement, ils sont caractérisés par l'adoption d'une allure de préservation de la relation. La relation et son maintien ont « une valeur intrinsèque ; 
corrélativement, les parties ont l'obligation d'ajuster leurs prestations de manière à ce que la relation contractuelle soit aussi satisfaisante que possible ${ }^{21}$.

Les contrats relationnels mettent en évidence le rôle de la réputation, de la répétition des relations contractuelles et d'autres facteurs dans la bonne gestion des contrats, surtout ceux qui sont liés aux affaires.

Par contre, les syndicats, les associations, les centres de recherche indépendants, les universités sont des organisations caractérisées par l'assemblage de personnes physiques ou morales qui ont des intérêts convergents et à qui convient le maintien du lien contractuel. Différemment d'une société commerciale, ces organisations n'ont pas le partage de la rentabilité comme leur objet, mais des actions qui bénéficieront leurs associés, cela veut dire que la mesure de la satisfaction est dans le cadre de l'individualité. Actions collectives dirigées au bénéfice individuel.

Que ce soit des syndicats (et leurs catégories professionnelles), une association (et ses intérêts analogues), un centre de recherche indépendant (et les impacts sociaux et économiques des produits qui seront offerts au marché), une université (et la demande d'attention des autorités publiques par rapport à la formation professionnelle, au développement technologique et aux produits), sont des exemples d'organisations relationnelles qui peuvent avoir un rôle important pour l'établissement de liens stables, basés sur des intérêts individuels qui seront objets d'un effort collectif, dans une ambiance de collaboration stimulée par l'importance accordée à la réputation.

À partir de la définition de ces organisations comme une des sources où la seule source de financements liés à la vie politique, c'est l'argent des associés qui sera versé aux partis politiques à partir d'une décision stratégique prise au sein de l'organisation. Je pense que cela peut bien attirer l'attention des associés et des parties prenantes aux besoins de participation effective à l'action politique, d'une façon organisée.

Quand nous avons adopté comme point de départ le choix relativement à savoir qui sera le donateur des sources de financement des campagnes électorales, nous avons proposé que ce soit celui à qui sera moins difficile la récupération (réelle ou potentielle) de la donation. Dans l'univers des organisations syndicales, associatives, de recherches scientifiques, de remboursement ou de compensation (qui peut être ou pas économique) sont assez clairs.

21 BOUTHINON-DUMAS, H. LES CONTRATS RELATIONNELS ET LA THEORIE DE L'IMPREVISION. REVUE INTERNATIONALE DE DROIT ECONOMIQUE, 2001/3 (T. XV, 3), P. 339-373. DOI : 10.3917/RIDE.153.0339. [HTTPS://WWW.CAIRN.INFO/REVUEINTERNATIONALE-DE-DROIT-ECONOMIQUE-2001-3.HTM-PAGE-339.HTM] (CONSULTE LE 9 DECEMBRE 2018). 


\section{CONCLUSION}

Quand ont été présentés les premières idées à propos de notre proposition au IMODEV, qui a eu lieu à Paris, au mois de novembre 2018, le sujet a provoqué une discussion très éclairante pour nous.

Une jeune fille a demandé si les mêmes défauts qui mettent en chèque le système actuel, la capture de l'autorité administrative étant une possibilité, la corruption et la prévalence des intérêts des petits groupes plus organisés et riches, d'autres possibilités, n'auraient pas par conséquent la neutralisation des bénéfices du système proposé.

À l'occasion nous avons répondu qu'il $\mathrm{n}$ y a pas une alternative au système démocratique électoral, ce qui veut dire qu'il va probablement être gardé comme option d'organisation de l'État, mais son modèle actuel est épuisé. Même si on considère que - en raison du comportement opportuniste ou des inégalités informationnelles - le modèle proposé sera victime des défauts équivalents, au moins PENDANT une certaine période il pourra générer des résultats plus satisfaisants que les résultats actuels.

Pour le moment, nous n'avons pas changé notre idée.

\section{BIBLIOGRAFIE}

AXELROD, ROBERT. The Evolution of Cooperation. New York, Basic Books, 1984.

BACKES, A. L. Financiamento Partidário e Eleitoral: Alemanha, França, Portugal e Espanha. Congrès National. Assessorie Legislative. 2013, [http: / /www2.camara.leg.br/atividadelegislativa/estudos-e-notas-tecnicas/publicacoes-da-consultoria-legislativa/areas-daconle/tema3/2012_22272.pdf] (consulté le 10 octobre 2018).

BOURCIER, N. ; GATINOIS, C. Brésil : tout comprendre à l'opération « Lava Jato ». 2017. www.lemonde.fr/ameriques/article/2017/03/26/affaire-petrobras-retour-sur-les-trois-anneesqui-ont-marque-le-bresil_5100932_3222.html].

BOUTHINON-DUMAS, H. Les contrats relationnels et la théorie de l'imprévision. Revue internationale de droit économique, 2001/3 (t. XV, 3), p. 339-373. DOI : 
10.3917/ride.153.0339. [https://www.cairn.info/revue-internationale-de-droit-economique2001-3.htm-page-339.htm] (Consulté le 9 decembre 2018)

COASE, Ronald. The Problem of Social Cost. Journal of Law and Economics, Vol. 3 (Oct., 1960), pp. 1-44, Published by: The University of Chicago Press Stable [http://www.jstor.org/stable/724810] (consulté le 12 novembre 2018).

COASE, Ronald ; DOWNS, A. Uma Teoria Econômica da Democracia, São Paulo, Edusp, 1999.

KANAYAMA, Rodrigo. O mito do eleitor racional. in : Análise Econômica do Direito: Justiça e Desenvolvimento. M. C. P. RIBEIRO, V. H. DOMINGUES, V. KLEIN, Vinícius (dir.), Curitiba, Editora CRV, 2016, pp.23-26.

LOLOUP, L. Blockchain: La revolution de la confiance. Paris, Eyrolles, 2018.

LESPAGNOL, J. L'influence de l'Internet sur les candidats. In : L'Internet et la démocratie numérique [en ligne]. Perpignan : Presses universitaires de Perpignan, 2016 (généré le 06 décembre 2018). [http://books.openedition.org/pupvd/2770]. ISBN : 9782354122980. DOI : 10.4000/books. pupvd.2770 (consulté le 2 décembre 2018).

NORTH, D. C. Economic performance through time. In: The American Economic Review, 1994, p. 359-368.

OECD(2017). Le financement de la démocratie: Financement des partis politiques et des campagnes électorales et risque de capture de l'action publique. Examens de l'OCDE sur la gouvernance publique, OECD Publishing, Paris. p. 31. https://doi.org/10.1787/9789264263994fr.

SEN, Amartya. Desenvolvimento como liberdade. 4. éd. , São Paulo, Companhia das Letras, 2010.

TAPSCOTT, D.' ; TAPSCOTT, A. Blockchain Revolution: How the technology behind bitcoin and others cryptocurrencies is changing the word. New York, Portfolio/Penguin, 2018.

WILLIAMSON, Oliver. The economic institutions of capitalism: Firms, Markets, Relational Contracting, New York, The Free Press, 1985.

ZINGALES, Luigi. Um capitalismo para o povo: reencontrando a chave da prosperidade americana, São Paulo, BEI Comunicação, 2015. 


\section{COMO FAZER REFERÊNCIA AO ARTIGO (ABNT):}

ALVES, Giovani Ribeiro Rodrigues; RIBEIRO, Marcia Carla Pereira. Le financement des campagnes politiques: un dialogue avec la theorie d'Oliver Williamson. Revista Eletrônica do Curso de Direito da UFSM, Santa Maria, RS, v. 15, n. 3, e39511, set./dez. 2020. ISSN 1981-3694. DOI: http://dx.doi.org/10.5902/1981369439511. Disponível em: https://periodicos.ufsm.br/revistadireito/article/view/39511 Acesso em: dia mês. ano.

Direitos autorais 2020 Revista Eletrônica do Curso de Direito da UFSM

Editores responsáveis: Rafael Santos de Oliveira e Angela Araujo da Silveira Espindola

\section{cc) $(1) \ominus$ BY NO ND}

Esta obra está licenciada com uma Licença Creative Commons Atribuição-NãoComercial-SemDerivações 4.0 Internacional.

\section{SOBRE OS AUTORES}

GIOVANI RIBEIRo RodRIGUES ALVES

Doutor e Mestre em Direito pela Universidade Federal do Paraná. Professor de Direito Empresarial de cursos de graduação e pós-graduação. Coordenador da Pós Graduação em Direito Empresarial Aplicado da FIEP. Autor de livro, artigos acadêmicos e capítulos de livros

\section{MARCia CARLa Pereira Ribeiro}

Possui graduação em Direito pela Universidade Federal do Paraná (1987), mestrado em Direito pela Universidade Federal do Paraná (1994) e doutorado em Direito pela Universidade Federal do Paraná (1998). Atualmente é professora titular da Pontifícia Universidade Católica do Paraná, professora associada da Universidade Federal do Paraná, ex-Procuradora Geral do Estado do Paraná. Foi professora visitante em estágio de pós-doutoramento na Escola de Direito de São Paulo da Fundação Getúlio Vargas e pesquisadora convidada da Université de Montréal. Tem pós doutorado pela Faculdade de Direito da Universidade de Lisboa e pela Universidade Paris 1 Sorbonne. Consultora da Fundação Araucária de Apoio ao Desenvolvimento Científico e Tecnológico do Paraná e é integrante de conselhos editoriais e consultivos de Revistas Especializadas. Pesquisadora de Produtividade da Fundação Araucária (2012/2016 e 2019...). Foi e Secretaria da Administração e da Previdência do Estado do Paraná (2016/0217). Tem experiência na área de Direito, com ênfase em Direito Comercial e Contratual, atuando principalmente nos seguintes temas: empresa, direito empresarial, direito falimentar, análise econômica do Direito e direito contratual. sociedades estatais. Governança e Gestão de riscos empresariais. Cooperativismo e Agronegócio. Ex-Presidente da ADEPAR Associação Paranaense de Direito e Economia (2009-2011) e da ABDE - Associação Brasileira de Direito e Economia (2010-2011). Integrante das câmaras de arbitragem da Associação Comercial do Paraná (ARBITAC) e da Federação das Indústrias do Paraná (CAMFIEP) 